\title{
Role of sfk1 Gene in the Filamentous Fungus Penicillium roqueforti
}

\author{
Claudia Torrent', Carlos Gil-Durán', Juan F. Rojas-Aedo', Exequiel Medina', \\ Inmaculada Vaca', Paulo Castro ${ }^{3}$, Ramón O. García-Rico ${ }^{4}$, Milena Cotoras', \\ Leonora Mendoza ${ }^{3}$, Gloria Levicán ${ }^{1}$ and Renato Chávez ${ }^{1 *}$ \\ ' Departamento de Biología, Facultad de Química y Biología, Universidad de Santiago de Chile, Santiago, Chile, \\ ${ }^{2}$ Departamento de Química, Facultad de Ciencias, Universidad de Chile, Santiago, Chile, ${ }^{3}$ Departamento de Química de los \\ Materiales, Facultad de Química y Biología, Universidad de Santiago de Chile, Santiago, Chile, ${ }^{4}$ GIMBIO Group, Department \\ of Microbiology, Faculty of Basic Sciences, Universidad de Pamplona, Pamplona, Colombia
}

The sfk1 (suppressor of four kinase) gene has been mainly studied in Saccharomyces cerevisiae, where it was shown to be involved in growth and thermal stress resistance. This gene is widely conserved within the phylum Ascomycota. Despite this, to date sfk 1 has not been studied in any filamentous fungus. Previously, we found that the orthologous of sfk 1 was differentially expressed in a strain of Penicillium roqueforti with an altered phenotype. In this work, we have performed a functional characterization of this gene by using RNAi-silencing technology. The silencing of $s f k 1$ in $P$. roqueforti resulted in decreased apical growth and the promotion of conidial germination, but interesting, it had no effect on conidiation. In addition, the attenuation of the sfk 1 expression sensitized the fungus to osmotic stress, but not to thermal stress. RNAmediated gene-silencing of sfk 1 also affected cell wall integrity in the fungus. Finally, the silencing of sfk 1 depleted the production of the main secondary metabolites of $P$. roqueforti, namely roquefortine $\mathrm{C}$, andrastin $\mathrm{A}$, and mycophenolic acid. To the best of our knowledge this is the first study of the sfk 1 gene in filamentous fungi.

Keywords: Penicillium roqueforti, suppressor of four kinase, RNA-mediated gene silencing, phenotypic changes, secondary metabolites

\section{INTRODUCTION}

Fungi use a complex network of signal transduction pathways to sense abiotic and biotic signals from the environment (Park et al., 2010). Therefore, signal transduction pathways have pivotal importance in fungi. Currently, signal transduction pathways in model fungi such as Saccharomyces cerevisiae and Aspergillus spp. have been widely studied. Conversely, these pathways have been scarcely studied in other species such as the filamentous fungus Penicillium roqueforti. $P$. roqueforti is important to the food industry, particularly in the production of several kind of ripened blue-veined cheeses (Fernández-Bodega et al., 2009). In addition, this fungus produces several interesting secondary metabolites (García-Estrada and Martín, 2016). Despite this, cellular processes in $P$. roqueforti have been poorly studied.

In $P$. roqueforti, the most studied signal transduction pathway corresponds to the heterotrimeric G proteins, particularly the $\alpha$-subunit from the subgroup I (G $\alpha \mathrm{i})$. By overexpressing a dominant active G $\alpha$ i subunit, it has been demonstrated that this protein influences several processes in the fungus. Thus, this protein negatively affects growth, sporulation, and thermal and osmotic stress resistance in P. roqueforti (García-Rico et al., 2007, 2008a, 2009). Furthermore, Gai stimulates 
germination and increases the production of the secondary metabolite roquefortine C (García-Rico et al., 2009).

$\mathrm{G} \alpha \mathrm{i}$ signaling produces pleiotropic effects on fungal cells. Therefore, Gai could be affecting several other regulators. In P. roqueforti, Gai signaling negatively affects the expression of $p c z 1$, a gene encoding for a $\mathrm{Zn}(\mathrm{II})_{2} \mathrm{Cys}_{6}$ protein (Gil-Durán et al., 2015). Interestingly, $p c z 1$ showed a positive effect on apical extension and conidiation, whereas it had negative effect on conidial germination (Gil-Durán et al., 2015).

To date, few molecular determinants involved in the control of cellular processes in P. roqueforti have been described. With the aim of unveiling genes from $P$. roqueforti that could be related with Gai signaling, in a previous work we performed suppression subtractive hybridization (SSH) experiments (GilDurán et al., 2015). From these experiments, several cDNA sequences differentially expressed were obtained, including a full cDNA corresponding to the orthologous of the $s f k 1$ (suppressor of four kinase) gene from S. cerevisiae.

The sfkl gene was originally discovered in S. cerevisiae, and encodes for a transmembrane protein (Sfk1) located at the plasma membrane, whose specific function is the proper localization of a phosphatidylinositol 4-kinase named Stt4 to the membrane (Audhya and Emr, 2002). Stt4 is an enzyme involved in the phosphoinositide second messengers pathway that regulate several cellular processes in the yeast (Audhya et al., 2000), and to fulfill its role, Stt4 must be tethered to the plasma membrane by Sfk1 (Voelker, 2005).

A preliminary inspection of GenBank database records indicates that the orthologs of Sfk 1 are widely conserved within the phylum Ascomycota (unpublished data), but strikingly, to date this gene has not been studied in filamentous fungi. Therefore, we decide to characterize this gene in $P$. roqueforti.

\section{MATERIALS AND METHODS}

\section{Fungal Strain and Culture Media}

The wild-type strain P. roqueforti CECT 2905 (ATCC 10110) was used in this work. The strain was routinely kept on Power medium agar (Fierro et al., 1996). Other media used in this work were Czapek minimal medium, YES agar (Rojas-Aedo et al., 2017) and CM liquid medium (Gil-Durán et al., 2015).

\section{Construction of Plasmid pSfk1-RNAi for sfk1 Silencing and Transformation of $P$. roqueforti CECT 2905}

To generate the $s f k 1$ knockdown construct, plasmid pJL43-RNAi (Ullán et al., 2008) was used. This plasmid has two promoters in opposite directions, and an $\mathrm{NcoI}$ site between the promoters (Ullán et al., 2008).

A 450-bp fragment of the sfk1 gene was amplified with primers RiSfkF (5' - AGACTCCCATGGCCACTGGACTATGAT CG - $3^{\prime}$ ) and RiSfkR (5'- AGACTCCCATGGAACGACAATGAA CGAGGA - $3^{\prime}$ ). Both primers contain an NcoI site (underlined). The amplified product was digested with $\mathrm{NcoI}$ and ligated into pJL43-RNAi (also previously digested with $\mathrm{NcoI}$ and dephosphorylated), giving rise to plasmid pSfk1-RNAi. The pSfk1-RNAi plasmid was confirmed by sequencing (data not shown) and used to transform $P$. roqueforti CECT 2905 according to the protocol described by Gil-Durán et al. (2015). In addition, a control strain that contains the empty plasmid pJL43-RNAi was obtained.

\section{Nucleic Acids Extractions and RT-PCR Assays}

DNA and total RNA from $P$. roqueforti were extracted as described by Gil-Durán et al. (2015). RT-PCR experiments were performed as described by García-Rico et al. (2017). Briefly, total RNA extracted was treated with RNase-free DNase I (Roche, Germany) and then quantified using a $\mu$ Drop plate in a MultiSkan GO quantification system (Thermo Scientific, Germany). To synthesize sfkl cDNA, RevertAid Reverse Transcriptase (Thermo Scientific, Germany) was used according the manufacturer' instructions. Reactions contained $200 \mathrm{ng}$ of treated RNA and $10 \mathrm{pmol}$ of primers RiSfkF and RiSfkR. Amplification of $\beta$-tubulin cDNA (internal control) was performed as described by García-Rico et al. (2017). The products of the reactions were resolved by electrophoresis and quantified by densitometry analysis using the "myImageAnalysis" software (Thermo Scientific, Germany). Values were expressed as percentage of the ratio between $s f k 1 / \beta$-tubulin.

\section{Phylogenetic Analysis}

The deduced sequence of Sfk1 protein from $P$. roqueforti CECT 2905 was used to find similar sequences using BlastP. Sequences obtained were used to perform phylogenetic analysis. Briefly, sequences were aligned by Clustal Omega and the phylogenetic trees were reconstructed using the Neighbor-joining method with the MEGA version 7 program (Kumar et al., 2016). Evolutionary distances data were calculated using the Poisson correction model. The support of internal nodes was performed by bootstrap analyses with 1,000 replications.

\section{Phenotypic Characterization, Analysis of Thermal and Hypertonic Stress Resistance, and Cell Wall Integrity Assay}

The apical extension rates, the production of conidia, and the measurements of conidial germination were done exactly as described by Gil-Durán et al. (2015). The thermal and hypertonic stress analyses were performed as described by García-Rico et al. (2017).

The cell wall integrity assays were performed using calcofluor white as described by Mendoza et al. (2016). Briefly, conidia from $P$. roqueforti at a final concentration of $1 \times 10^{4}$ conidia/mL were inoculated in 24-well plates (lined with 12 -mm glass coverslips) containing $1 \mathrm{~mL}$ of $\mathrm{CM}$ medium. Cultures were incubated at $28^{\circ} \mathrm{C}$ for $15 \mathrm{~h}$ until the germination of conidia. After this time, the medium was removed and glass coverslips with $P$. roqueforti hyphae adhered were washed three times with $0.9 \% \mathrm{NaCl}$, and stained with calcofluor white during $10 \mathrm{~min}$. Then the glass coverslips containing hyphae were washed again and mounted on slides. The fluorescence of $P$. roquerfot $i$ 
hyphae stained with calcofluor white was observed under a fluorescence microscope with Blue/Violet (110033V2) filter. The fluorescence intensity was quantified using the program "myImageAnalysis" v2.0 (Thermo Scientific, Germany). For this purpose, the fluorescence intensity of hyphae from three randomly-chosen fields of each sample was recorded. The average of the fluorescence intensities recorded from the wild-type strain was set as $100 \%$ of fluorescence. The rest of the samples were normalized with respect to the wild-type strain. As control of cell wall damage, germinated conidia from the wild-type strain were treated with Lysing Enzymes from Trichoderma harzianum $(20 \mathrm{mg} / \mathrm{mL})$ during $2 \mathrm{~h}$ at $28^{\circ} \mathrm{C}$, and stained with calcofluor white as above.

\section{Extraction of Secondary Metabolites and HPLC Analyses}

The production, extraction and HPLC analyses of mycophenolic acid and andrastin A from $P$. roqueforti CECT 2905 have been described elsewhere (Del-Cid et al., 2016; Rojas-Aedo et al., 2017). In the case of roquefortine $C$, the strains were grown on YES agar for 15 days at $28^{\circ} \mathrm{C}$. The mycelia were collected, suspended in a chloroform-methanol (2:1) mixture, and then submitted to sonication during $30 \mathrm{~min}$. The mixture was filtered through nylon membranes (mesh $30 \mu \mathrm{m}$ ). The extracted mycelia were kept for further dry-weight determination (see below), whereas the filtrated was evaporated to dryness in a rotary evaporator. Previous to HPLC analysis, the dried filtrated was suspended in $500 \mu \mathrm{l}$ of methanol (HPLC grade) and briefly centrifuged. Twenty $\mu \mathrm{l}$ of this suspension were submitted to HPLC analysis. HPLC analysis was performed using a Waters 1525 HPLC equipment (Waters, Ireland) with a $4.6 \mathrm{~mm} \times 250 \mathrm{~mm}(5 \mu \mathrm{m})$ SunFire C18 reverse phase column. The column was held at $35^{\circ} \mathrm{C}$. The mobile phase was water with $0.02 \%$ TFA (solvent A) and acetonitrile with $0.02 \%$ TFA (solvent B). The gradient program was as follows: $15 \% \mathrm{~B}$ to $47 \%$ B linear over $15 \mathrm{~min}, 47 \% \mathrm{~B}$ to $100 \% \mathrm{~B}$ over $1 \mathrm{~min}$, and isocratic $5 \mathrm{~min}$. The flow rate used was $1.2 \mathrm{~mL} / \mathrm{min}$. For the detection and quantification of the compound, pure roquefortine C (Santa Cruz Biotechnology) was used as standard. The detection of roquefortine $\mathrm{C}$ was performed at $254 \mathrm{~nm}$. Under the conditions described, roquefortine $\mathrm{C}$ showed a retention time of $10.9 \mathrm{~min}$. Roquefortine $\mathrm{C}$ production was normalized by dry weight of fungal mycelia. For this purpose, the mycelium from each sample was dried as described by García-Rico et al. (2009).

\section{Nucleotide Sequence Accession Number}

The nucleotide sequence of the $s f k 1$ gene described in this work has been deposited in the GenBank database under accession number MF614690.

\section{RESULTS}

\section{Analysis of sfk1 Sequence}

In a previous work, we performed SSH experiments to looking for genes from $P$. roqueforti CECT 2905 that are regulated by Gai (Gil-Durán et al., 2015). Among the sequences obtained from these experiments, a full cDNA matching with hypothetical $s f k 1$ genes from ascomycetous fungi (mainly Penicillium species) was obtained. This cDNA sequence is 914 nt- long, excluding the poly-A tail. By using genomic DNA from $P$. roqueforti CECT 2905 and suitable primers (designed from the cDNA sequence), we amplified the $s f k 1$ gene by PCR. The gene was sequenced and compared with the cDNA sequence, revealing the presence of four introns (data not shown). The $s f k 1$ gene from strain CECT 2905 was also submitted to BlastN analysis against the genome of $P$. roqueforti FM164 (Cheeseman et al., 2014). We found that $s f k 1$ gene from $P$. roqueforti CECT 2905 exactly matches (100\% identity, 100\% coverage, and no gaps) with the $s f k 1$ gene from strain FM164, located at contig ProqFM164S01-10 in the genome sequence of this strain (data not shown).

The $s f k 1$ gene from $P$. roqueforti CECT 2905 encodes a protein of 235 amino acids. BlastP analysis of the deduced protein revealed the presence of orthologs in a wide range of species of filamentous fungi from the phylum Ascomycota, mainly from the genera Penicillium, Aspergillus, and Talaromyces. Using several of these sequences, a phylogenetic analysis was performed (Figure 1A). This analysis revealed that Sfk1 sequences are clustered in agreement with the expected evolutionary relationship among these genera. On the other hand, using all Sfk1 sequences from Penicillium species retrieved by BlastP (22 sequences including Sfk1 from $P$. roqueforti), a second phylogenetic analysis was performed (Figure 1B). Eighteen sequences (including Sfk1 from $P$. roqueforti) belong to species from the subgenus Penicillium, whereas five sequences belong to species from the subgenus Aspergilloides (Figure 1B). In this case, the Sfk1 sequences also are clustered in agreement with the expected evolutionary relationship among these Penicillium species (Figure 1B).

Finally, and according to bioinformatics predictions (TMHMM Server v. 2.0 and TMpred at Expasy), the Sfk1 protein from $P$. roqueforti is a transmembrane protein. It contains six putative transmembrane helix domains, with its amino and carboxyl ends inside oriented (data not shown).

\section{RNA-Mediated Gene-Silencing of $s f k 1$}

RNA-mediated gene silencing was used to perform the functional characterization of $s f k 1$ in $P$. roqueforti CECT 2905. The fungus was transformed with pSfk1-RNAi, and 57 phleomycin-resistant transformants were obtained. Fifteen transformants were randomly selected and subjected to RTPCR (see section "Materials and Methods"). Two of these transformants (hereafter SFK19 and SFK38) showed a reduction of $s f k 1$ transcripts of approximately 55\% compared to the wild-type strain (Figure 2A). In addition, the presence of silencing cassette in these transformants was also confirmed by PCR (Figure 2B). Taken together, all these data indicate the successful silencing of $s f k 1$ in transformants SFK19 and SFK38. 


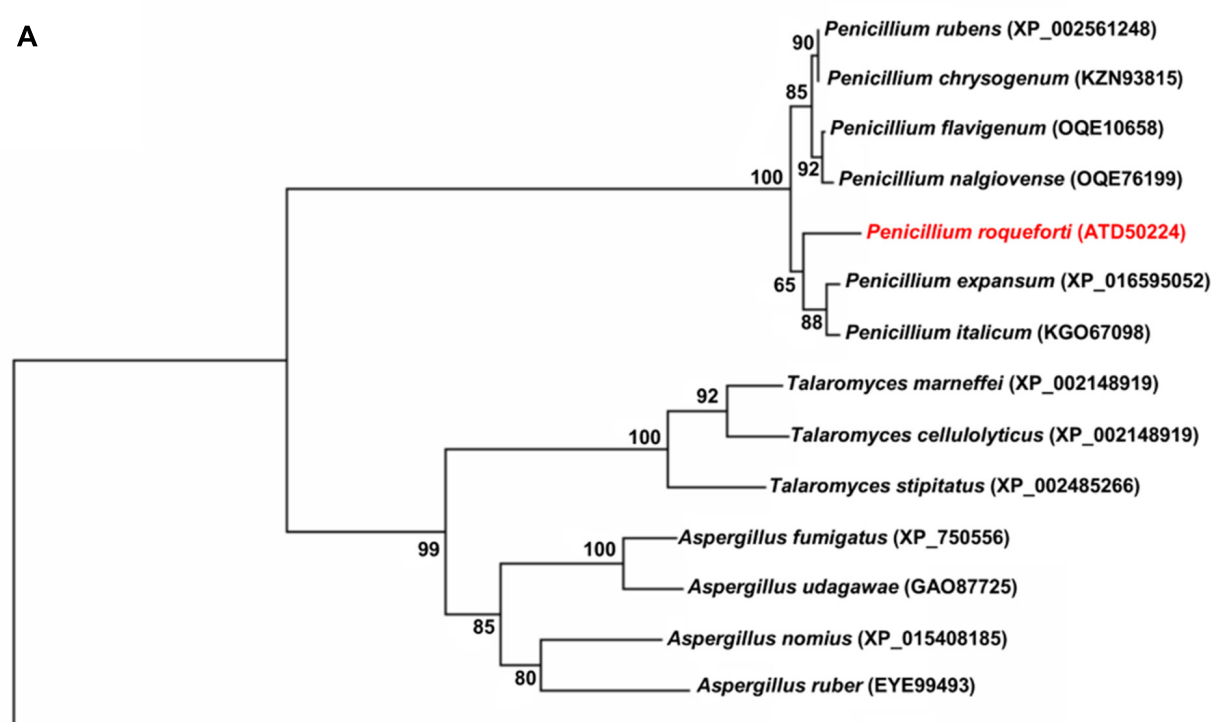

Saccharomyces cerevisiae (KZV09890)

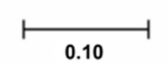

B

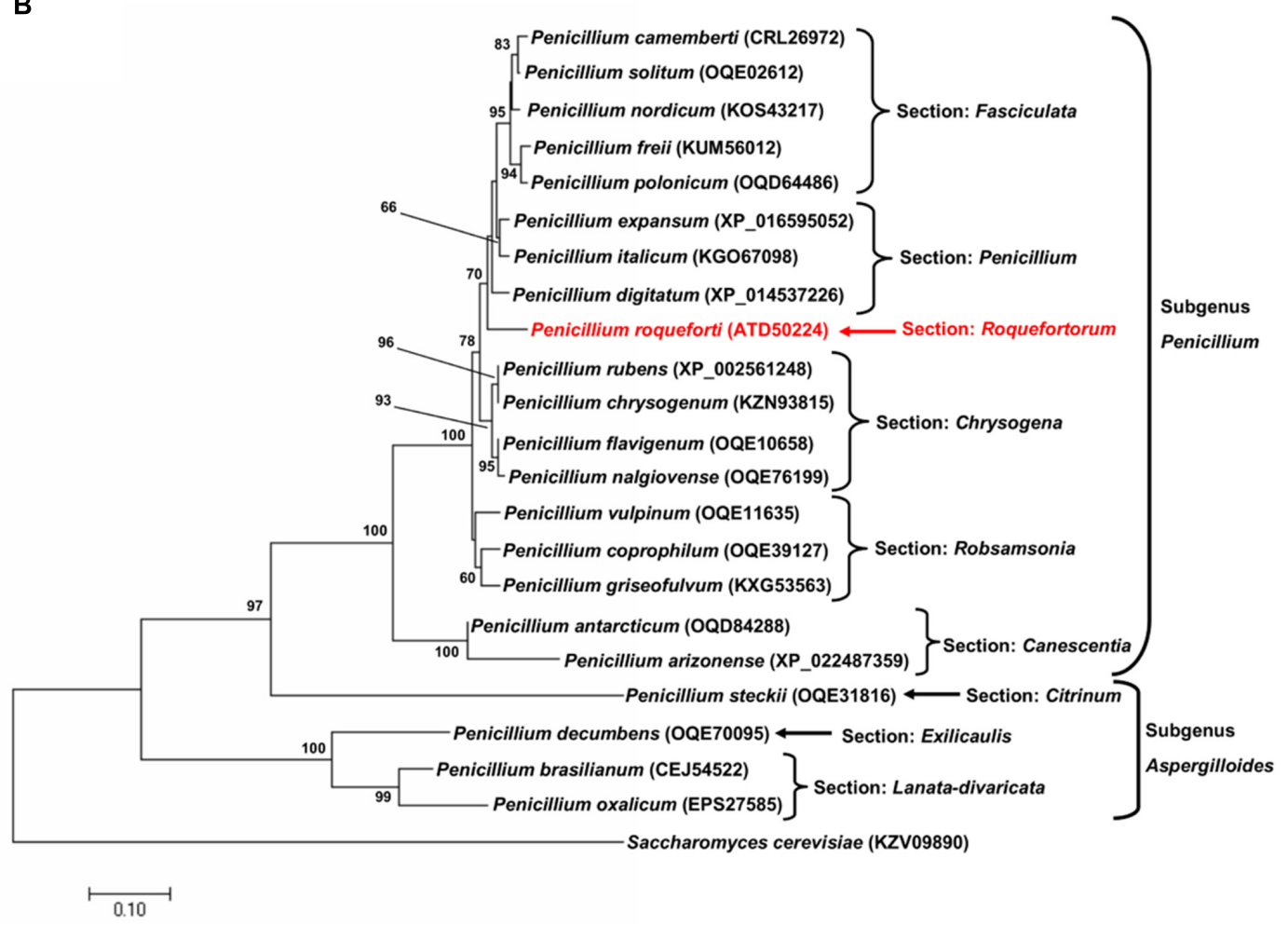

FIGURE 1 | Evolutionary relationships of Sfk1 from Penicillium roqueforti CECT 2905 and its orthologous proteins. (A) Evolutionary tree of Sfk1 proteins from species from the genera Penicillium, Aspergillus, and Talaromyces. The sequence from P. roqueforti is highlighted in red. In parenthesis, the accession number of each sequence is indicated. Sfk1 from Saccharomyces cerevisiae was used as outgroup. Bootstrap values (50\% or higher) are shown in each node. (B) Evolutionary tree of all Sfk1 proteins from the genus Penicillium retrieved from BlastP analysis. The sequences are present in Penicillium species assigned to different sections within the subgenera Penicillium or Aspergilloides. The sequence from P. roqueforti is highlighted in red. In parenthesis, the accession number of each protein sequence is indicated. Sfk1 from S. cerevisiae was used as outgroup. Bootstrap values (50\% or higher) are shown in each node. 


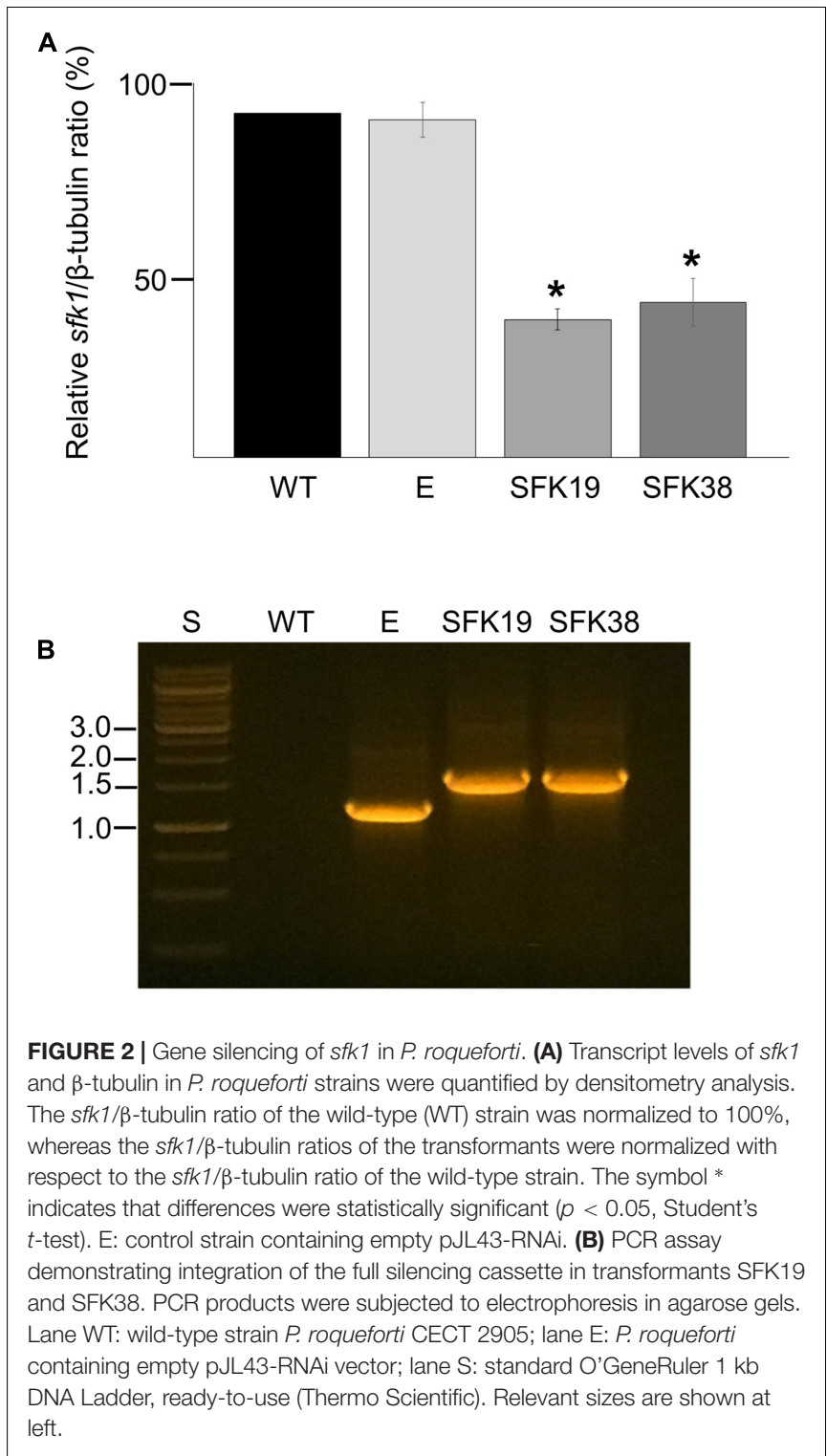

\section{The Knockdown of sfk1 Decreases Growth Rate and Increases Conidial Germination, But Does Not Affect Conidiation in $P$. roqueforti}

Since the deletion of $s f k 1$ produces changes in the growth pattern of S. cerevisiae (Jin et al., 2008), we analyzed the effect of the RNA-mediated silencing of $s f k 1$ on growth in $P$. roqueforti. Figure 3A shows that the colonies of the transformants SFK19 and SFK38 grown on Czapek minimal medium or Power rich medium were smaller than the wild-type strain, suggesting that the knockdown of $s f k 1$ altered growth of $P$. roqueforti. Figure 3B shows the apical extension rates of transformants SFK19 and SFK38 on the same media. As observed, and independently from the medium used, the attenuation of $s f k 1$ altered normal growth of the fungus. On Czapek medium, the apical growth rate of strains SFK19 and SFK38 ranged between 0.170 and
$0.185 \mathrm{~mm} / \mathrm{h}$, versus $0.240 \mathrm{~mm} / \mathrm{h}$ in the wild-type strain, whereas on Power medium, the apical growth rate of strains SFK19 and SFK38 ranged between 0.20 and $0.22 \mathrm{~mm} / \mathrm{h}$, versus $0.31 \mathrm{~mm} / \mathrm{h}$ in the wild-type strain (Figure 3B). These results indicate that on Czapek medium, the transformants grew at a rate between 72 and $77 \%$ of wild-type strain levels. Similarly, on Power medium the transformants grew at a rate between 64 and $75 \%$ of wild-type strain levels. Therefore, we conclude that the knockdown of $s f k 1$ in $P$. roqueforti caused a reduction in the apical extension rate.

We also tested conidia production in all the strains. Interestingly, we found that the RNA-mediated silencing of sfkl does not affect conidiation (Figure 4A). However, the knockdown of $s f k 1$ affected conidial germination. Figure 4B shows that all the strains follow a sigmoidal pattern of conidial germination, but in transformants SFK19 and SFK38, this process was earlier (around $1 \mathrm{~h}$ ) compared with the wild-type strain. For example, at $9 \mathrm{~h}$, around $20 \%$ of conidia from transformants have already germinated, whereas the wild-type strain reaches the same percentage of germination at $10 \mathrm{~h}$. Similar differences are observed along all the exponential phase of germination (Figure 4B).

\section{The Knockdown of sfk1 Decreases Hypertonic Stress Resistance But Does Not Affect Heat Shock Resistance in $P$. roqueforti}

In S. cerevisiae, Sfk1 is important for response to heat shock (Audhya and Emr, 2002). For this reason, we tested thermal stress resistance of $P$. roqueforti strains. Remarkably, we did not found any difference in the ability to resist heat shock between the wildtype strain and transformants SFK19 and SFK38 (Figure 5A). On the other hand, we also tested hypertonic stress resistance, and we found interesting differences. Thus, the RNA-mediated silencing of $s f k 1$ decreased osmotic stress resistance to $\mathrm{NaCl}$ or $\mathrm{KCl}$ (Figure 5B). As average, the relative apical extension rate of transformants SFK 19 and SFK38 was approximately $66 \%$ of wild-type levels in the presence of $\mathrm{KCl}$ or $\mathrm{NaCl}$.

\section{The RNA-Mediated Gene-Silencing of sfk1 Affects Cell Wall Integrity in $P$. roqueforti}

It has been suggested that $S f k 1$ could be important for cell wall integrity (see section "Discussion"), so we analyzed the effect of the RNA-mediated silencing of $s f k 1$ on the cell wall integrity in $P$. roqueforti by using calcofluor white (Figure 6). This compound is a fluorochrome molecule which binds to the chitin in the cell walls of the fungi. Therefore, a decrease in cell wall fluorescence intensity produced by calcofluor white is indicative of cell wall damage (Mendoza et al., 2016). Our results indicate that compared to the wild-type strain, transformants SFK19 and SFK38 have lower fluorescence intensity (Figure 6A). In the case of strain SFK19, it shows approximately $74 \%$ of the fluorescence of wild-type strain levels, whereas in SFK38 the effect is more dramatic, because this strain shows approximately $45 \%$ of the fluorescence of wild-type strain levels (Figure 6B). These data suggest that the knockdown of $s f k 1$ 


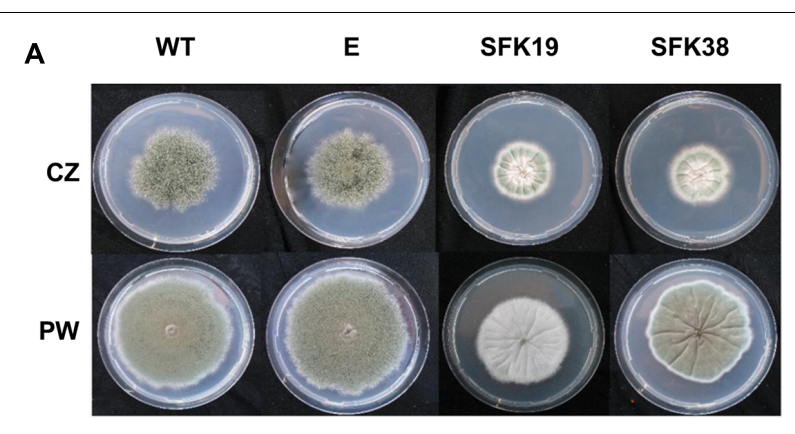

B

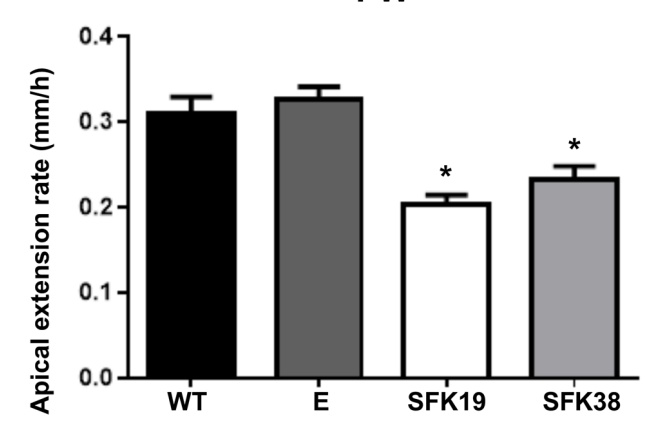

CZ

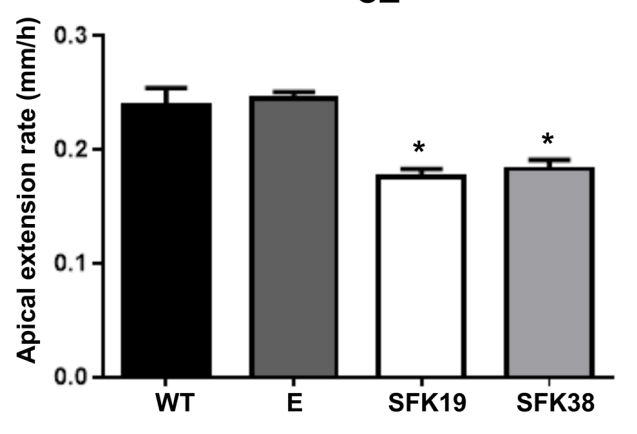

FIGURE 3 | Effect of RNA-mediated gene-silencing of sfk1 on growth of P. roqueforti. (A) Phenotypes of P. roqueforti CECT 2905 (WT) and transformants SFK19 and SFK38. The colonies were grown for 7 days at $28^{\circ} \mathrm{C}$ in poor Czapek (CZ) or rich Power (PW) media. Note the reduction in growth of strains SFK19 and SFK38 as compared to the wild-type strain, especially in Czapek medium. P. roqueforti containing empty pJL43-RNAi vector (E) is included as control. This strain was undistinguishable from the wild-type strain. (B) Comparison of apical extension rates $(\mathrm{mm} / \mathrm{h})$ of $P$. roqueforti strains on Power (PW) and Czapek (CZ) media. Error bars represent standard deviation of three replicas from three different experiments. The symbol * indicates that differences were statistically significant $(p<0.05$, Student's $t$-test).

affected the cell wall integrity in transformants SFK19 and SFK38.

\section{The Knockdown of sfk1 Decreases the Production of the Secondary Metabolites Roquefortine C, Mycophenolic Acid, and Andrastin A in $P$. roqueforti}

Roquefortine C, mycophenolic acid, and andrastin A are some of the main secondary metabolites produced by P. roqueforti (García-Estrada and Martín, 2016), so we studied if RNA-mediated silencing of $s f k 1$ affected their production. As shown in Figure 7 the production of these secondary metabolites is depleted in transformants SFK19 and SFK38 compared to the wild-type strain. In the case of andrastin $\mathrm{A}$, the wild-type strain produces approximately $900 \mu \mathrm{g} / \mathrm{mg}$ of the compound, whereas in the same conditions, the transformants SFK19 and SFK38 produce approximately $250 \mu \mathrm{g} / \mathrm{mg}$ (Figure 7B). More evident is the effect on roquefotine C. In this case, the wild-type strain produces approximately $560 \mu \mathrm{g} / \mathrm{mg}$, whereas the transformants produce barely $15 \mu \mathrm{g} / \mathrm{mg}$ (Figure 7B). Finally, the most drastic effect was observed for mycophenolic acid. The wild-type strain produces approximately $300 \mu \mathrm{g} / \mathrm{mg}$ of this compound, but is almost undetectable in transformants SFK19 and SFK38 (Figure 7B). These results strongly suggest that the knockdown of $s f k 1$ decreases the production of the main secondary metabolites from P. roqueforti.

\section{DISCUSSION}

In $S$. cerevisiae, the overexpression of $s f k 1$ produces an interesting phenotypic change: different to the usual isotropic growth, yeasts overexpressing $s f k 1$ show filamentous-like growth phenotype (Jin et al., 2008). On the other hand, and by using overexpression and deletion mutants, it was demonstrated that $s f k 1$ has a positive effect in the thermal resistance in S. cerevisiae (Audhya and Emr, 2002). Further, the $s f k 1$ gene is overexpressed when S. cerevisiae is exposed to toxic substances such as benzene (North et al., 2011), trichothecenes (Suzuki and Iwahashi, 2012) and copper (Hodgins-Davis et al., 2012), suggesting that this gene could be related to the stress response in the yeast.

RNA-mediated silencing of $s f k 1$ reduced apical growth in $P$. roqueforti (Figure 3). This result matches well with those obtained in $S$. cerevisiae. In the yeast, the elimination of $s f k 1$ produced smaller colonies compared with the wild-type strain (Audhya and Emr, 2002), whereas the overexpression of the gene resulted in a filamentous-like growth (Jin et al., 2008). Although further work is necessary to extend the observations made in $P$. roqueforti to other filamentous fungi, our results and the previous results obtained in S. cerevisiae suggest that 


\section{CZ}

A

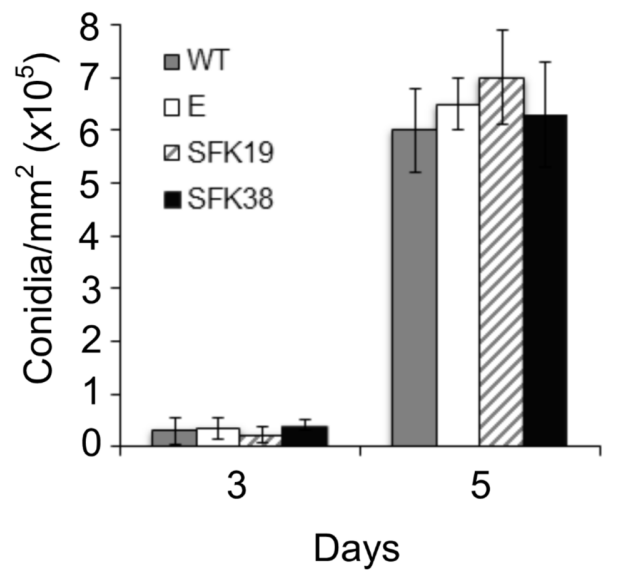

PW

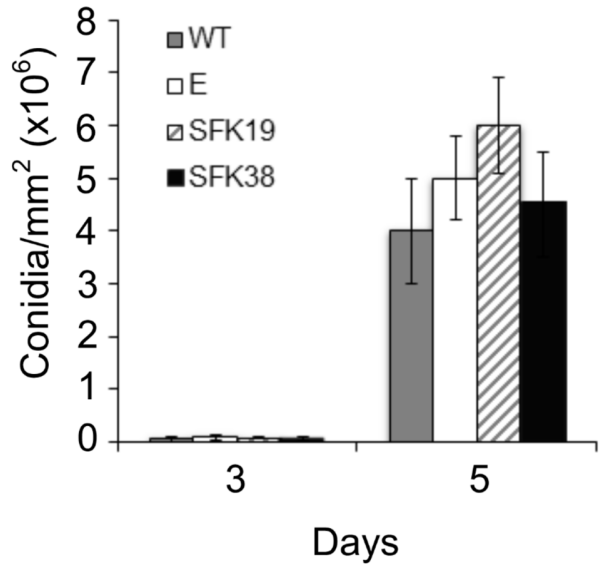

B

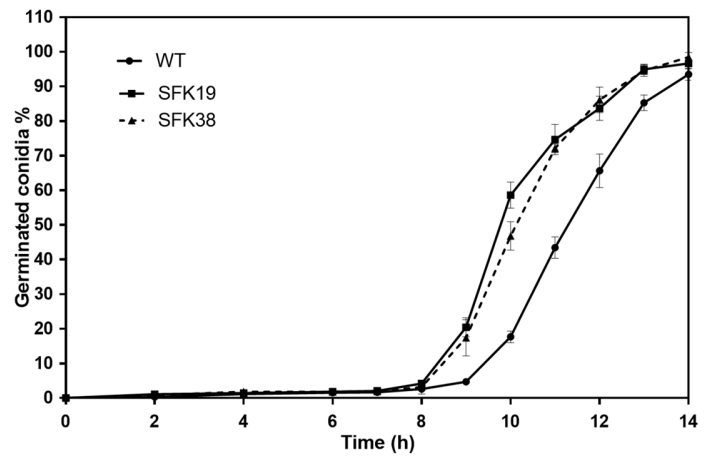

FIGURE 4 | Effect of RNA-mediated gene-silencing of sfk 1 on conidiation and conidial germination of $P$. roqueforti. (A) Conidial production by P. roqueforti CECT 2905 and transformants SFK19 and SFK38. $5 \times 10^{4}$ conidia were seeded in Petri dishes containing Czapek (CZ) or Power (PW) media. Dishes were incubated at $28^{\circ} \mathrm{C}$ for 3 or 5 days, and the conidia produced were collected and counted as described by Gil-Durán et al. (2015). Values are expressed as conidia/mm² of surface. A strain containing empty pJL43-RNAi vector (E) was used as a control. Error bars represent the standard deviation of three replicas from three independent experiments. The differences in conidial production of strains SFK19 and SFK38 were statistically undistinguishable $(p<0.05$ using Student's $t$-test) as compared to the wild-type and control strains. (B) Germination kinetics of $P$. roqueforti CECT 2905 (WT) and transformants SFK19 and SFK38. Conidia from each strain were inoculated in $\mathrm{CM}$ liquid media and incubated at $28^{\circ} \mathrm{C}$. At regular intervals, samples were taken, and the number of germinated and non-germinated conidia was counted under the microscope in 10 randomly-chosen fields. Data were expressed as the percentage of germinated conidia vs. hours of incubation. Error bars represent the standard deviation of three replicates in three independent experiments. No differences were observed between the wild-type strain and $P$. roqueforti containing empty pJL43-RNAi vector (data not shown).

$s f k 1$ is a positive regulator of vegetative growth. In yeasts, it was demonstrated that Sfk1 stabilizes Stt4 at the plasma membrane (Audhya and Emr, 2002). Stt4 is a phosphatidylinositol 4-kinase involved in the production of phosphatidylinositol-4-phosphate (PI4P) and in turn, PI4P activates PKC pathway producing actin organization (Audhya and Emr, 2002). Accordingly, it can be hypothesized that the RNA-mediated silencing of $s f k 1$ in $P$. roqueforti resulted in an impaired organization of actin. Taking into account that actin has a pivotal role during polarized hyphal growth (Steinberg et al., 2017), the impaired organization of actin produced by the reduction of $s f k 1$ transcripts can explain the reduced apical growth observed in $P$. roqueforti. Further work is necessary to demonstrate this proposal.

According to our results, $s f k 1$ does not affect conidiation. In $P$. roquefort $i$, it has been proposed the existence of a dual control of conidiation by Gai through the cAMP-dependent and CAMPindependent pathways (García-Rico et al., 2008a). In addition, recent evidence suggests that $p c z 1$, probably through cAMPindependent pathway, stimulates conidiation in this fungus through positive regulation of the expression of conidiationspecific genes $b r l A$, wetA, and $a b a A$ (Gil-Durán et al., 2015). Our results suggest that $s f k 1$ would not be involved in any of these pathways regulating conidiation.

Our results also indicate that $s f k 1$ has a negative role in conidial germination. Spore germination is triggered by environmental factors, which are sensed and transduced by signal transduction pathways that have been studied in detail in few species (Fillinger et al., 2002; Doehlemann et al., 2006; Krijgsheld et al., 2013). As in other fungi, P. roqueforti $\mathrm{G} \alpha$ i signaling has been demonstrated to control conidial germination (García-Rico et al., 2009) and recently it was suggested that $p c z 1$ may be participating in this Gai-signaling pathway controlling conidial germination (Gil-Durán et al., 2015). Here we show for the first time that $s f k 1$ is also involved in the regulation of conidial germination. As 

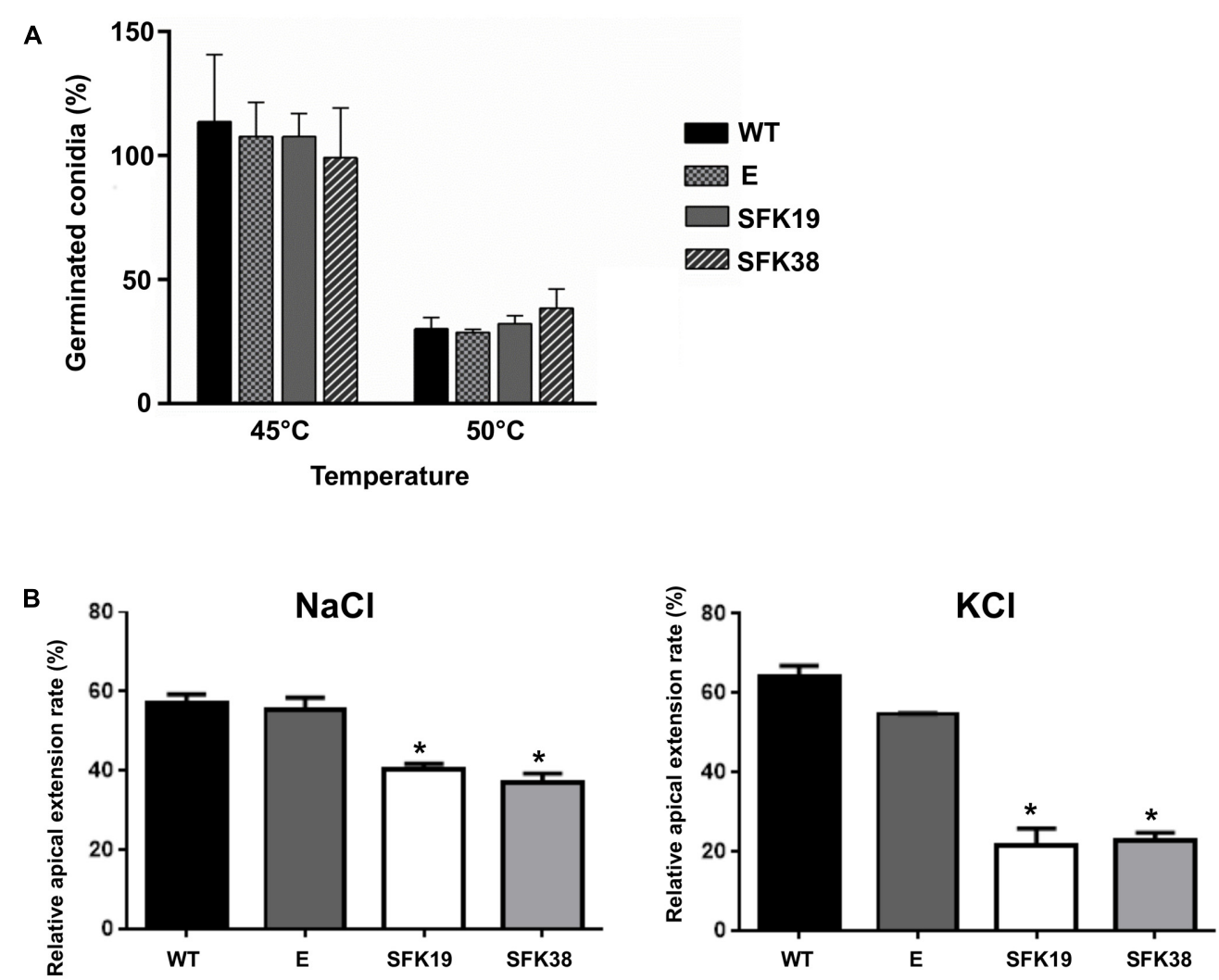

FIGURE 5 | Analysis of thermal hypertonic stress resistance of $P$. roqueforti CECT 2905 and transformants SFK19 and SFK38. (A) Percentage of viable conidia after $1 \mathrm{~h}$ of heat shock treatments at 45 and $50^{\circ} \mathrm{C}$. E: control strain containing empty pJL43-RNAi. Error bars represent the standard deviation of three replicas from three independent experiments. The differences in conidial production of strains SFK19 and SFK38 were statistically undistinguishable ( $0<0.05$ using Student's $t$-test) as compared to the wild-type and control strains. (B) Relative apical growth rates in Czapek minimal containing $0.45 \mathrm{M} \mathrm{NaCl}$ or $0.7 \mathrm{M} \mathrm{KCl}$. Error bars represent the standard deviation of three replicas from three independent experiments. The symbol * indicates that reductions in stress resistance of strains SFK19 and SFK38 were statistically significant ( $p<0.05$ using Student's $t$-test) as compared to the wild-type strain. E: control strain containing empty pJL43-RNAi.

deduced, the regulation of conidial germination in $P$. roqueforti is unclear, and more work is required to know how $s f k 1$ contributes to the control of spore germination process.

In $S$. cerevisiae, $s f k 1$ changes its expression pattern when the yeast is exposed to different toxic substances (North et al., 2011; Hodgins-Davis et al., 2012; Suzuki and Iwahashi, 2012), suggesting that this gene would be a general stress response determinant. Beyond this, its role in specific stressing conditions has not been fully evaluated and understood. Here, we found that the RNA-mediated silencing of $s f k 1$ reduced osmotic stress resistance in $P$. roqueforti (Figure 5). To the best of our knowledge, this is the first report describing the involvement of $s f k 1$ in osmotic stress response in fungi. Fungi sense osmotic stress through the HogA-MAPK pathway (Bahn et al., 2007; Hagiwara et al., 2016). Interestingly, a recent study has indirectly related HogA-MAPK pathway with Sfk1. Specifically, during their investigation about the role of Torl on hyphal elongation in Candida albicans, Su et al. (2013) found that: (i) Sfk1 may be a Tor1 regulator or perform a function in the Tor1 pathway; (ii) Tor1 signaling downregulates Hog1 basal activity. Accordingly, and assuming that these regulators are likely conserved in $P$. roqueforti, it can be hypothesized that the RNA-mediated silencing of $s f k 1$ reduced osmotic stress resistance in $P$. roquefort $i$ through the HogA-MAPK pathway. Further studies are needed to demonstrate this interesting hypothesis.

Audhya and Emr (2002) demonstrated that Sfk1 is a positive regulator of thermal stress resistance in S. cerevisiae. However, we did not found differences in thermal stress resistance between $P$. roqueforti wild-type and the SFK19 and SFK38 transformants. Mechanisms of thermal stress resistance in fungi are multifactorial and not fully understood. Several reports have showed that fungi regulate thermal stress response through the same HogA-MAPK pathway described above (Alonso-Monge et al., 2009; Ji et al., 2012). Further, as specific responses to elevated temperature, fungi modify membrane composition and produce intracellular molecules that act as thermal protectants, such as heat shock proteins and trehalose (Leach and Cowen, 2014; Hagiwara et al., 2016). Our results suggest that different to $S$. cerevisiae, $s f k 1$ would not influence on these response mechanisms in $P$. roqueforti, so the thermal stress resistance pathway in $P$. roqueforti could be somewhat different from yeast.

In $S$. cerevisiae, Stt4 is required for cell wall integrity (Audhya et al., 2000). In turn, Stt4 is tethered to the plasma membrane by Sfk1 (Voelker, 2005). These evidences suggest 

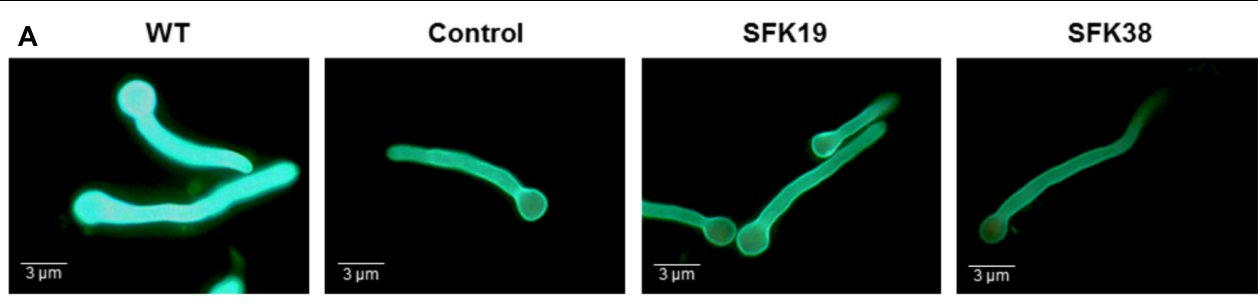

B

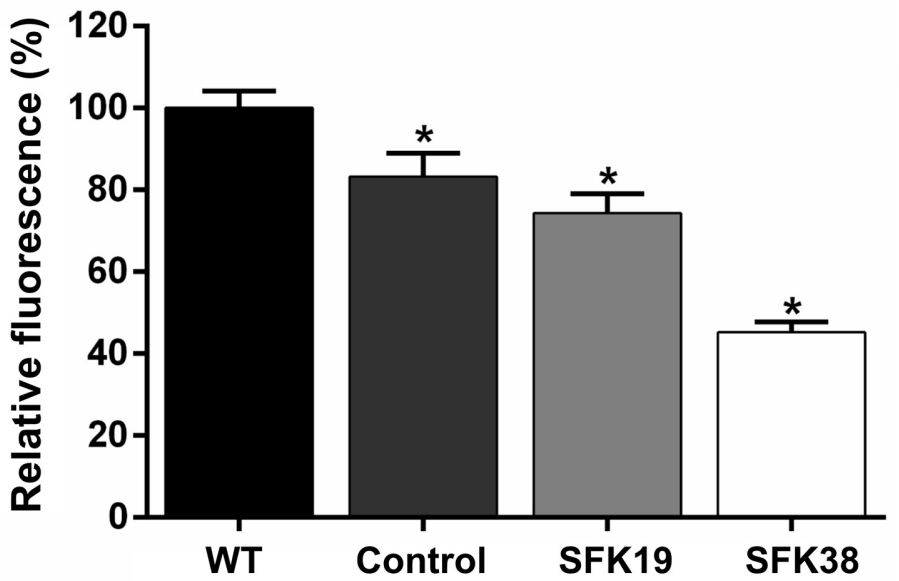

FIGURE 6 | Effect of the sfk1 gene on the cell wall integrity of P. roqueforti CECT 2905 (WT) and transformants SFK19 and SFK38. (A) Representative images of germinated conidia after calcofluor staining. Note the decreased fluorescence in transformants SFK19 and SFK38 as compared to the wild-type strain. As control of cell wall damage, P. roqueforti CECT 2905 was treated with "Lysing enzymes" from T. harzianum, as described in Section "Materials and Methods." (B) Data were quantified and expressed as percentage of relative fluorescence, as described in Section "Materials and Methods." The symbol * indicates that reductions in intensity of fluorescence were statistically significant $(p<0.05$ using Student's $t$-test) as compared to the wild-type strain.
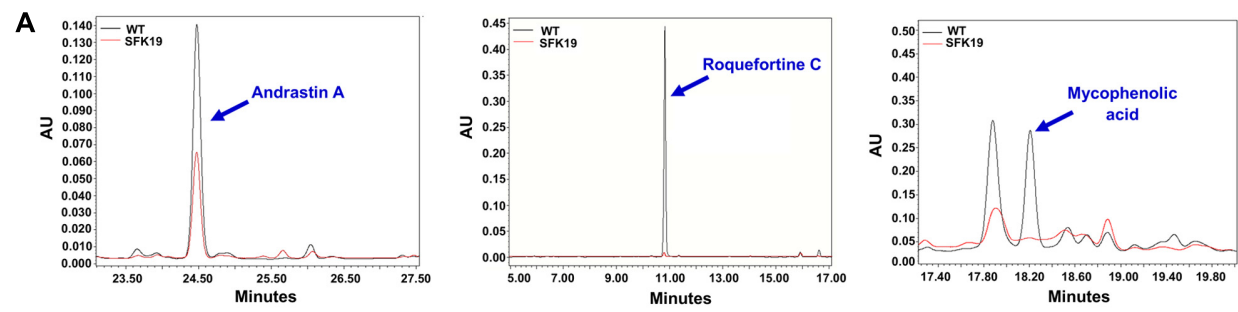

B
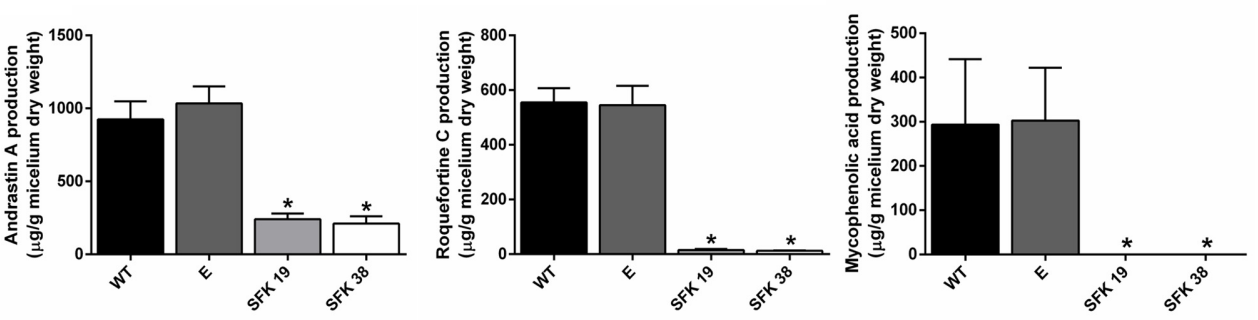

FIGURE 7 | Production of secondary metabolites andrastin A, roquefortine C, and mycophenolic acid by P. roqueforti CECT 2905 and transformants SFK19 and SFK38. (A) Examples of HPLC trace chromatograms (300 nm) of the transformant SFK19 (red line) as compared to the wild-type strain (black line). In each panel, the peak representing the specific compound is indicated by a blue arrow. Note the reduction of the peaks in transformant SFK19 as compared to the wild-type strain. The same behavior was observed in transformant SFK30 (not shown). (B) Metabolites extracted were quantified and normalized by the dry weight of the fungal mycelia as described in Section "Materials and Methods." Left panel: andrastin A. Center panel: roquefortine C. Right panel: mycophenolic acid. Error bars represent the standard deviation of three replicates in three independent experiments. Statistical analysis (Student's $t$-test, $p<0.05$ ) indicates significant differences between the production of the secondary metabolite by the wild-type strain and the transformants $\left.{ }^{*}\right)$. E: control strain containing empty pJL43-RNAi. 
that Sfk1, indirectly, could be important in maintenance of cell wall integrity (Audhya and Emr, 2002; Levin, 2005). Our results support this prediction, because the RNA-mediated silencing of $s f k 1$ reduced cell wall integrity in $P$. roquefort $i$ (Figure 6). However, how exactly $s f k 1$ affects cell wall integrity in fungi is unknown. To the best of our knowledge, at least two putative mechanisms have been proposed: (i) the direct recruitment/activation of effector proteins by PI4P (generated by Stt4) and the downstream metabolite phosphatidylinositol-4,5biphosphate; (ii) the activation of effectors through the MAPKcell wall integrity (CWI) pathway (Audhya and Emr, 2002; Levin, 2005).

The RNA-mediated silencing of $s f k 1$ drastically reduced the production of andrastin $\mathrm{A}$, roquefortine $\mathrm{C}$ and mycophenolic acid in $P$. roqueforti (Figure 7 ). Although the enzymatic pathways for the biosynthesis of these secondary metabolites in $P$. roqueforti have been recently elucidated (Kosalková et al., 2015; Del-Cid et al., 2016; Rojas-Aedo et al., 2017), the cellular mechanisms controlling their biosynthesis are not fully understood. As example, none of the cluster for the biosynthesis of these compounds contains a gene with similarity to a putative transcription factor (Kosalková et al., 2015; DelCid et al., 2016; Rojas-Aedo et al., 2017), suggesting that their biosynthesis would be controlled by global regulators. In the case of roquefortine $\mathrm{C}$, studies performed in different Penicillium species ( $P$. roqueforti, $P$. chrysogenum, and $P$. decumbens) indicate that the production of this metabolite could be controlled by $\mathrm{G} \alpha \mathrm{I}$ protein and the regulator of conidiation BrlA (García-Rico et al., 2008b, 2009; Qin et al., 2013) but remarkably, its biosynthesis would not depend on LaeA (Kosalková et al., 2009), a global regulator that controls secondary metabolism in several fungi. Regarding mycophenolic acid

\section{REFERENCES}

Alonso-Monge, R., Román, E., Arana, D. M., Pla, J., and Nombela, C. (2009). Fungi sensing environmental stress. Clin. Microbiol. Infect. 15, 17-19. doi: 10.1111/j. 1469-0691.2008.02690.x

Audhya, A., Foti, M., and Emr, S. D. (2000). Distinct roles for the yeast phosphatidylinositol 4-kinases, Stt4p and Piklp, in secretion, cell growth, and organelle membrane dynamics. Mol. Biol. Cell 11, 2673-2689. doi: 10.1091/mbc. 11.8.2673

Audhya, A., and Emr, S. D. (2002). Stt4 PI 4-kinase localizes to the plasma membrane and functions in the Pkcl-mediated MAP kinase cascade. Dev. Cell 2, 593-605. doi: 10.1016/S1534-5807(02)00168-5

Bahn, Y. S., Xue, C., Idnurm, A., Rutherford, J. C., Heitman, J., and Cardenas, M. E. (2007). Sensing the environment: lessons from fungi. Nat. Rev. Microbiol. 5, 57-69. doi: 10.1038/nrmicro1578

Cheeseman, K., Ropars, J., Renault, P., Dupont, J., Gouzy, J., Branca, A., et al. (2014). Multiple recent horizontal transfers of a large genomic region in cheese making fungi. Nat. Commun. 5:2876. doi: 10.1038/ncomms3876

Del-Cid, A., Gil-Durán, C., Vaca, I., Rojas-Aedo, J. F., García-Rico, R. O., Levicán, G., et al. (2016). Identification and functional analysis of the mycophenolic acid gene cluster of Penicillium roqueforti. PLOS ONE 11:e0147047. doi: 10.1371/journal.pone.0147047

Doehlemann, G., Berndt, P., and Hahn, M. (2006). Different signalling pathways involving a $\mathrm{G} \alpha$ protein, cAMP and a MAP kinase control germination of Botrytis cinerea conidia. Mol. Microbiol. 59, 821-835. doi: 10.1111/j.1365-2958. 2005.04991.x and andrastin $\mathrm{A}$, to the best of our knowledge regulators controlling their biosynthesis have not been described yet. Therefore, our finding that $s f k 1$ regulates the biosynthesis of roquefortine $\mathrm{C}$, mycophenolic acid and andrastin $\mathrm{A}$ is an important step forward in understanding the processes that regulate the biosynthesis of secondary metabolites in P. roqueforti.

\section{AUTHOR CONTRIBUTIONS}

IV, RG-R, MC, LM, GL, and RC conceived and designed the experiments, contributed reagents/materials, analyzed the data and supervised work. CT, CG-D, JR-A, EM, IV, and PC carried out the experiments and analyzed the data. EM and $\mathrm{RC}$ performed bioinformatics analysis. IV and RC drafted the manuscript. All authors have read and approved the manuscript.

\section{FUNDING}

This work received funds from grants Fondecyt 1120833 and Proyectos Basal USA 1555-VRIDEI 021743CR_PUBLIC, Universidad de Santiago de Chile. CG-D and JR-A have received doctoral fellowships CONICYTPFCHA/DoctoradoNacional/2013-21130251 and CONICYTPFCHA/Doctorado Nacional/2014-63140056, respectively.

\section{ACKNOWLEDGMENT}

The support of VRIDEI-USACH is acknowledged.

Fernández-Bodega, M. A., Mauriz, E., Gómez, A., and Martín, J. F. (2009). Proteolytic activity, mycotoxins and andrastin a in Penicillium roqueforti strains isolated from cabrales, valdeón and bejes-tresviso local varieties of blue-veined cheeses. Int. J. Food Microbiol. 136, 18-25. doi: 10.1016/j.ijfoodmicro.2009. 09.014

Fierro, F., Montenegro, E., Gutiérrez, S., and Martín, J. F. (1996). Mutants blocked in penicillin biosynthesis show a deletion of the entire penicillin gene cluster at a specific site within a conserved hexanucleotide sequence. Appl. Microbiol. Biotechnol. 44, 597-604. doi: 10.1007/BF00172491

Fillinger, S., Chaveroche, M. K., Shimizu, K., Keller, N., and d'Enfert, C. (2002). cAMP and ras signalling independently control spore germination in the filamentous fungus Aspergillus nidulans. Mol. Microbiol. 44, 1001-1016. doi: 10.1046/j.1365-2958.2002.02933.x

García-Estrada, C., and Martín, J. F. (2016). Biosynthetic gene clusters for relevant secondary metabolites produced by Penicillium roqueforti in blue cheeses. Appl. Microbiol. Biotechnol. 100, 8303-8313. doi: 10.1007/s00253-016-7788-x

García-Rico, R. O., Martín, J. F., and Fierro, F. (2007). The pgal gene of Penicillium chrysogenum NRRL 1951 encodes a heterotrimeric G protein alpha subunit that controls growth and development. Res. Microbiol. 158, 437-446. doi: 10.1016/j. resmic.2007.03.001

García-Rico, R. O., Fierro, F., and Martín, J. F. (2008a). Heterotrimeric G $\alpha$ protein pgal of Penicillium chrysogenum controls conidiation mainly by a cAMP-independent mechanism. Biochem. Cell Biol. 86, 57-69. doi: 10.1139/ O07-148

García-Rico, R. O., Fierro, F., Mauriz, E., Gómez, A., Fernández-Bodega, M. A., and Martín, J. F. (2008b). The heterotrimeric $\mathrm{G} \alpha$ protein pgal regulates biosynthesis 
of penicillin, chrysogenin and roquefortine in Penicillium chrysogenum. Microbiology 154, 3567-3578. doi: 10.1099/mic.0.2008/019091-0

García-Rico, R., Chávez, R., Fierro, F., and Martín, J. F. (2009). Effect of a heterotrimeric $\mathrm{G}$ protein $\alpha$ subunit on conidia germination, stress response, and roquefortine $C$ production in Penicillium roqueforti. Int. Microbiol. 12, 123-129.

García-Rico, R., Gil-Durán, C., Rojas-Aedo, J. F., Vaca, I., Figueroa, L., Levicán, G., et al. (2017). Heterotrimeric G protein alpha subunit controls growth, stress response, extracellular protease activity, and cyclopiazonic acid production in Penicillium camemberti. Fungal Biol. 121, 754-762 doi: 10.1016/j.funbio.2017. 05.007

Gil-Durán, C., Rojas-Aedo, J. F., Medina, E., Vaca, I., García-Rico, R. O., Villagrán, S., et al. (2015). The $p c z 1$ gene, which encodes a $\mathrm{Zn}(\mathrm{II}) 2 \mathrm{Cys} 6$ protein, is involved in the control of growth, conidiation, and conidial germination in the filamentous fungus Penicillium roqueforti. PLOS ONE 10:e0120740. doi: 10.1371/journal.pone. 0120740

Hagiwara, D., Sakamoto, K., Abe, K., and Gomi, K. (2016). Signaling pathways for stress responses and adaptation in Aspergillus species: stress biology in the post-genomic era. Biosci. Biotechnol. Biochem. 80, 1667-1680. doi: 10.1080/ 09168451.2016.1162085

Hodgins-Davis, A., Adomas, A. B., Warringer, J., and Townsend, J. P. (2012). Abundant gene-by-environment interactions in gene expression reaction norms to copper within Saccharomyces cerevisiae. Genome Biol. Evol. 4, 1061-1079. doi: 10.1093/gbe/evs084

Ji, Y., Yang, F., Ma, D., Zhang, J., Wan, Z., Liu, W., et al. (2012). HOG-MAPK signaling regulates the adaptive responses of Aspergillus fumigatus to thermal stress and other related stress. Mycopathologia 174, 273-282. doi: 10.1007/ s11046-012-9557-4

Jin, R., Dobry, C. J., McCown, P. J., and Kumar, A. (2008). Large-scale analysis of yeast filamentous growth by systematic gene disruption and overexpression. Mol. Biol. Cell 19, 284-296. doi: 10.1091/mbc.E07-05-0519

Kosalková, K., García-Estrada, C., Ullán, R. V., Godio, R. P., Feltrer, R., Teijeira, F., et al. (2009). The global regulator LaeA controls penicillin biosynthesis, pigmentation and sporulation, but not roquefortine $\mathrm{C}$ synthesis in Penicillium chrysogenum. Biochimie 91, 214-225. doi: 10.1016/j.biochi.2008.09.004

Kosalková, K., Domínguez-Santos, R., Coton, M., Coton, E., García-Estrada, C., Liras, P., et al. (2015). A natural short pathway synthesizes roquefortine $\mathrm{C}$ but not meleagrin in three different Penicillium roqueforti strains. Appl. Microbiol. Biotechnol. 99, 7601-7612. doi: 10.1007/s00253-015-6676-0

Krijgsheld, P., Bleichrodt, R., van Veluw, G. J., Wang, F., Müller, W. H., Dijksterhuis, J., et al. (2013). Development in Aspergillus. Stud. Mycol. 74, 1-29. doi: $10.3114 /$ sim0006

Kumar, S., Stecher, G., and Tamura, K. (2016). MEGA7: molecular evolutionary genetics analysis version 7.0 for bigger datasets. Mol. Biol. Evol. 33, 1870-1874. doi: 10.1093/molbev/msw054

Leach, M. D., and Cowen, L. E. (2014). To sense or die: mechanisms of temperature sensing in fungal pathogens. Curr. Fungal Infect. Rep. 8, 185-191. doi: 10.1007/ s12281-014-0182-1
Levin, D. E. (2005). Cell wall integrity signaling in Saccharomyces cerevisiae. Microbiol. Mol. Biol. Rev. 69, 262-291. doi: 10.1128/MMBR.69.2.262-291.2005

Mendoza, L., Castro, P., Melo, R., Campos, A. M., Zúñiga, G., Guerrero, J., et al. (2016). Improvement of the antifungal activity against Botrytis cinerea of syringic acid, a phenolic acid from grape pomace. J. Chil. Chem. Soc. 61, 3039-3042. doi: 10.4067/S0717-97072016000300006

North, M., Tandon, V. J., Thomas, R., Loguinov, A., Gerlovina, I., Hubbard, A. E., et al. (2011). Genome-wide functional profiling reveals genes required for tolerance to benzene metabolites in yeast. PLOS ONE 6:e24205. doi: 10.1371/ journal.pone. 0024205

Park, G., Jones, C. A., and Borkovich, K. A. (2010). "Signal transduction pathways," in Cellular and Molecular Biology of Filamentous Fungi, eds K. A. Borkovich, and D. J. Ebbole (Washington, DC: ASM Press), 50-59. doi: 10.1128/ 9781555816636.ch5

Qin, Y., Bao, L., Gao, M., Chen, M., Lei, Y., Liu, G., et al. (2013). Penicillium decumbens BrlA extensively regulates secondary metabolism and functionally associates with the expression of cellulase genes. Appl. Microbiol. Biotechnol. 97, 10453-10467. doi: 10.1007/s00253-013-5273-3

Rojas-Aedo, J. F., Gil-Durán, C., Del-Cid, A., Valdés, N., Álamos, P., Vaca, I., et al. (2017). The biosynthetic gene cluster for andrastin A in Penicillium roqueforti. Front. Microbiol. 8:813. doi: 10.3389/fmicb.2017.00813

Steinberg, G., Peñalva, M. A., Riquelme, M., Wösten, H. A., and Harris, S. D. (2017). Cell biology of hyphal growth. Microbiol. Spectr. 5:FUNK-0034-2016.

$\mathrm{Su}, \mathrm{C}$., Lu, Y., and Liu, H. (2013). Reduced TOR signaling sustains hyphal development in Candida albicans by lowering Hog1 basal activity. Mol. Biol. Cell 24, 385-397. doi: 10.1091/mbc.E12-06-0477

Suzuki, T., and Iwahashi, Y. (2012). Comprehensive gene expression analysis of type B trichothecenes. J. Agric. Food Chem. 60, 9519-9527. doi: 10.1021/ jf3020975

Ullán, R. V., Godio, R. P., Teijeira, F., Vaca, I., García-Estrada, C., Feltrer, R., et al. (2008). RNA-silencing in Penicillium chrysogenum and Acremonium chrysogenum: validation studies using $\beta$-lactam genes expression. J. Microbiol. Methods 75, 209-218. doi: 10.1016/j.mimet.2008.06.001

Voelker, D. R. (2005). Bridging gaps in phospholipid transport. Trends Biochem. Sci. 30, 396-404. doi: 10.1016/j.tibs.2005.05.008

Conflict of Interest Statement: The authors declare that the research was conducted in the absence of any commercial or financial relationships that could be construed as a potential conflict of interest.

Copyright (๑) 2017 Torrent, Gil-Durán, Rojas-Aedo, Medina, Vaca, Castro, GarcíaRico, Cotoras, Mendoza, Levicán and Chávez. This is an open-access article distributed under the terms of the Creative Commons Attribution License (CC BY). The use, distribution or reproduction in other forums is permitted, provided the original author(s) or licensor are credited and that the original publication in this journal is cited, in accordance with accepted academic practice. No use, distribution or reproduction is permitted which does not comply with these terms. 